Swarthmore College

Works

$5-1-2003$

\title{
Spheromak Merging And Field Reversed Configuration Formation At The Swarthmore Spheromak Experiment
}

C. D. Cothran

Abram Lockhart Falk , '03

Matthew J. Landreman , '03

Michael R. Brown

Swarthmore College, doc@swarthmore.edu

M. J. Schaffer

Follow this and additional works at: https://works.swarthmore.edu/fac-physics

Part of the Physics Commons

Let us know how access to these works benefits you

\section{Recommended Citation}

C. D. Cothran; Abram Lockhart Falk, '03; Matthew J. Landreman , '03; Michael R. Brown; and M. J. Schaffer. (2003). "Spheromak Merging And Field Reversed Configuration Formation At The Swarthmore Spheromak Experiment". Physics Of Plasmas. Volume 10, Issue 5. 1748-1754. DOI: 10.1063/1.1564084 https://works.swarthmore.edu/fac-physics/292

This work is brought to you for free by Swarthmore College Libraries' Works. It has been accepted for inclusion in Physics \& Astronomy Faculty Works by an authorized administrator of Works. For more information, please contact myworks@swarthmore.edu. 
Spheromak merging and field reversed configuration formation at the Swarthmore Spheromak Experiment

C. D. Cothran, A. Falk, A. Fefferman, M. Landreman, and M. R. BrownM. J. Schaffer

Citation: Physics of Plasmas 10, 1748 (2003); doi: 10.1063/1.1564084

View online: http://dx.doi.org/10.1063/1.1564084

View Table of Contents: http://aip.scitation.org/toc/php/10/5

Published by the American Institute of Physics

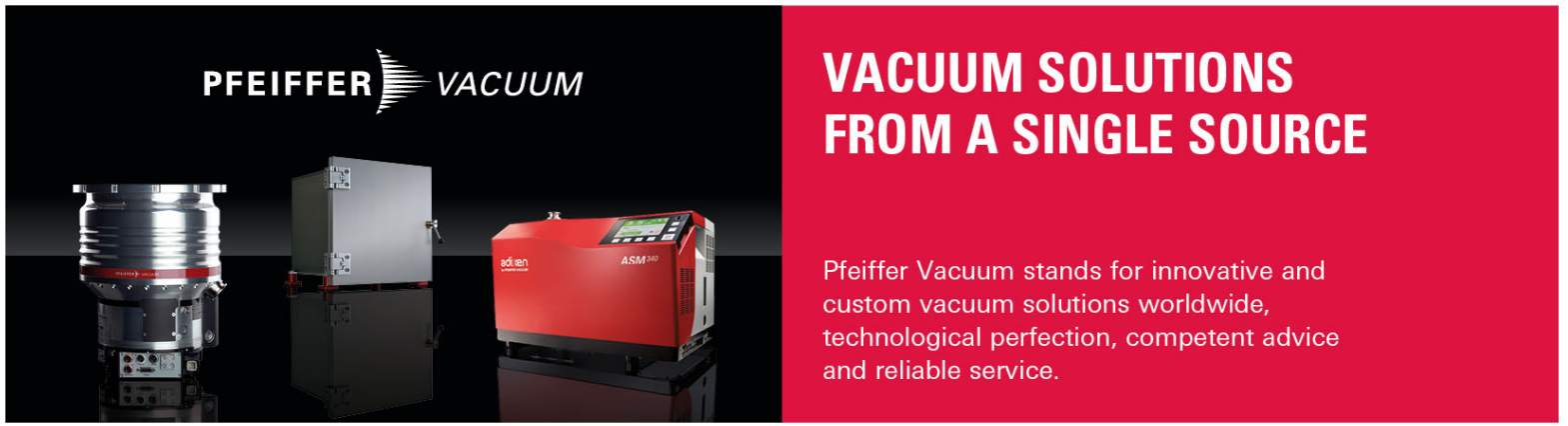




\title{
Spheromak merging and field reversed configuration formation at the Swarthmore Spheromak Experiment ${ }^{\text {a) }}$
}

\author{
C. D. Cothran, ${ }^{\text {b) }}$ A. Falk, A. Fefferman, M. Landreman, and M. R. Brown \\ Department of Physics and Astronomy, Swarthmore College, Swarthmore, Pennsylvania 19081 \\ M. J. Schaffer \\ General Atomics, San Diego, California 92186
}

(Received 15 November 2002; accepted 5 February 2003)

\begin{abstract}
The initial results of coaxial co- and counter-helicity spheromak merging studies at the Swarthmore Spheromak Experiment (SSX) [M. R. Brown, Phys. Plasmas 6, 1717 (1999)] are reported. In its new configuration, SSX is optimized to study field reversed configuration (FRC) formation and stability by counter-helicity spheromak merging. A pair of midplane coils magnetically restricts the merging process to determine how the stability of the resulting magnetic configuration depends upon the quantity of toroidal flux remaining from the initial spheromaks. The diagnostic set at SSX, featuring the capability of measuring up to 600 magnetic field components at $800 \mathrm{~ns}$ time resolution, permits detailed studies of the dynamic three-dimensional magnetic structures produced during these experiments. A compact array of magnetic probes examines the local reconnection process, while a distributed array of probes examines global magnetic structure. Counter-helicity merging produces a FRC that persists for several Alfvén times, although the oppositely directed toroidal field of the initial spheromaks does not completely annihilate. The $m=1$ (toroidal) mode dominates late in the evolution, and is consistent with the tilt instability. Co-helicity merging produces a single elongated spheromak that rapidly tilts. (C) 2003 American Institute of Physics. [DOI: 10.1063/1.1564084]
\end{abstract}

\section{INTRODUCTION}

The field reversed configuration (FRC) ${ }^{2}$ is a high $\beta$ compact toroidal (CT) plasma. In its idealized form, it has closed, purely poloidal field lines. These characteristics offer distinct advantages that make the FRC an extremely attractive fusion reactor concept. A reactor relevant FRC must be stable in the magnetohydrodynamic (MHD) fluid limit; often this is characterized by a large value of $s$, roughly given by the ratio of the minor radius to the ion gyroradius. Most experimental studies $^{3-5}$ show the FRC to be a stable configuration, albeit in the low $s$ (kinetic) regime, whereas various calculations, ${ }^{6-8}$ both kinetic and MHD based, predict instability, most notably to the tilt mode.

Theta-pinch formation, the method used for most FRC experiments, produces a fairly low flux, high temperature configuration. At present, these conditions makes the large $s$ regime difficult to reach. In experiments on the TS-3 device at the University of Tokyo, Ono ${ }^{9-11}$ pioneered FRC formation by counter-helicity spheromak merging. Reconnection annihilates the initial spheromak helicities, and the consumed magnetic energy is converted to ion thermal energy and flow. The TS-3 experiments demonstrated how reconnection directly accounts for the relaxation to a FRC, and, furthermore, that the relaxation bifurcates to a spheromak if the initial helicity imbalance is too great. ${ }^{12}$ Most importantly, this method can produce a FRC with more flux than can the theta-pinch, thus permitting studies at large $s$ at moderate temperatures.

\footnotetext{
${ }^{a)}$ Paper UI1 1, Bull. Am. Phys. Soc. 47, 321 (2002).

b)Invited speaker. Electronic mail: ccothra1@swarthmore.edu
}

The idealized FRC contains no toroidal field, but there have been experimental hints that toroidal fields can, in fact, be found in the FRC. Spheromak-like toroidal fields were observed in a translating FRC experiment. ${ }^{13}$ Of potentially greater interest is the axially antisymmetric distribution of toroidal field that naturally accompanies the counter-helicity spheromak merging process. While the TS-3 results showed an epoch where the initial toroidal fields were completely annihilated, the same distribution subsequently regenerated with opposite polarity. Recent numerical results ${ }^{14,15}$ from a large $s$ hybrid model demonstrated that poloidally sheared toroidal electron flow nonlinearly generates lobes of axially antisymmetric toroidal field. Moreover, the self-generated toroidal field suppressed the growth of the $m=1$ mode in this simulation. The TS-3 results unfortunately cannot assess this prediction because this device deliberately included a conducting column on the geometric axis to provide artificial stability to the $m=1$ tilt and shift modes.

Until recently, research at the Swarthmore Spheromak Experiment $(\mathrm{SSX})^{1}$ has focused on local studies of magnetic reconnection by partially merging counter-helicity spheromaks. ${ }^{16-18}$ Motivated by the TS-3 results, the program at SSX, now called SSX-FRC, has begun to examine the general issue of FRC stability at large $s$. The particular question of the stabilizing role of toroidal fields will be addressed by magnetically restricting the merging process with a set of midplane reconnection control coils (RCC) to limit the quantity of toroidal field annihilated from the initial counterhelicity spheromaks: stability as a function of the residual toroidal field strength can therefore be systematically investigated. The global, three-dimensional magnetic structure of 

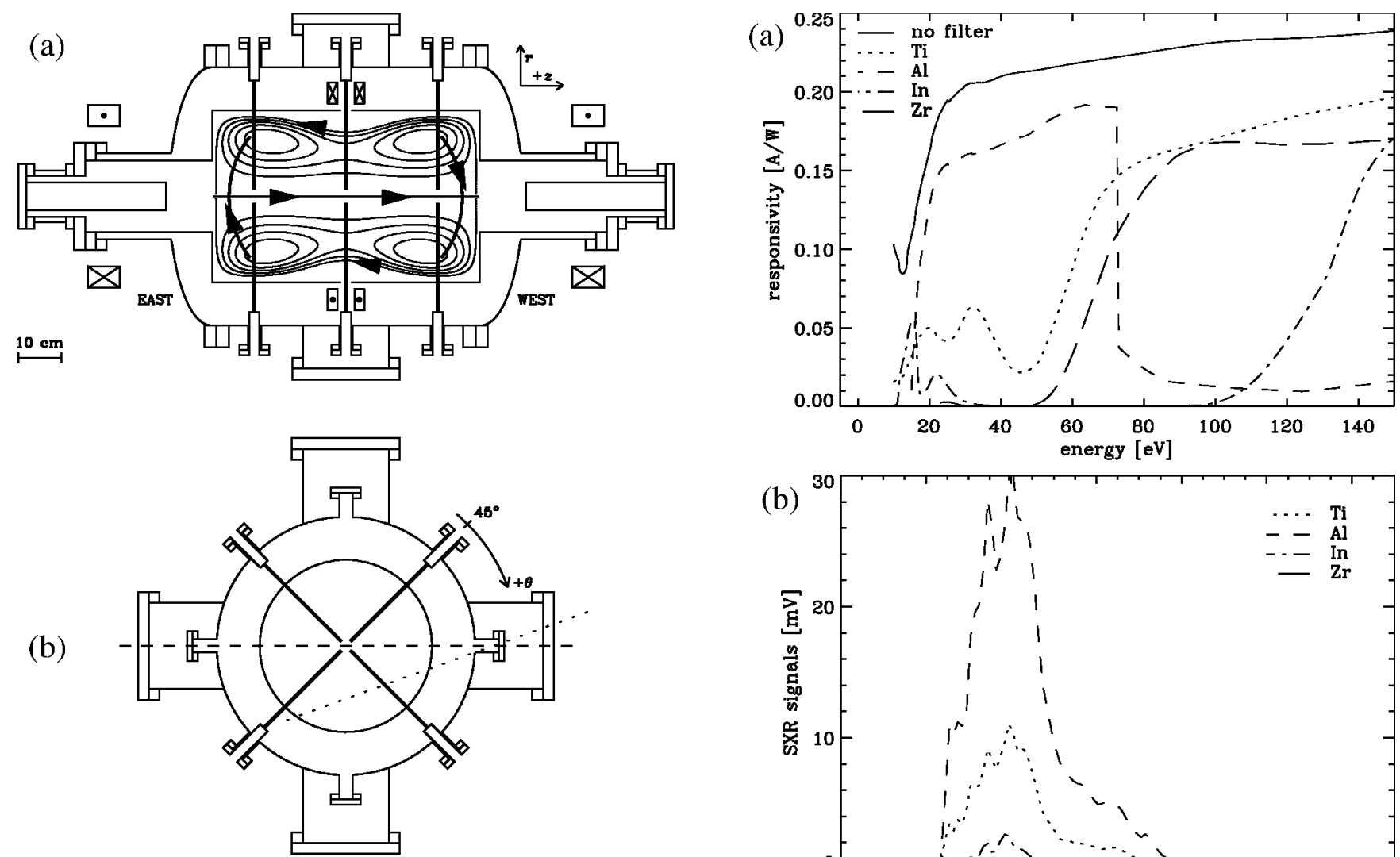

FIG. 1. A sketch of the SSX-FRC device. The flux conserver is $40 \mathrm{~cm}$ in diameter and $61 \mathrm{~cm}$ in length. Gun flux is typically $1 \mathrm{mWb}$. Reconnection control coils (RCC) can be programmed for field of up to $700 \mathrm{G}$ on axis. Up to 20 linear magnetic probes can be inserted. Each probe measures $\left(B_{r}, B_{\theta}, B_{z}\right)$ at eight radial positions. For this paper, a maximum of 12 probes were inserted, equally spaced in four toroidal angles in each of the three $z$ planes. Part (a) shows a poloidal flux surfaces from a numerical equilibrium with two magnetic axes (doublet CT) that might result from counter-helicity spheromak merging; the suggested toroidal field is also drawn. Included in part (b) are the lines of sight of the soft x-ray detector (dotted, midplane) and the interferometer (dashed, east side).

the configuration can be measured with up to 600 internal magnetic probes at $1.25 \mathrm{MHz}$ acquisition rate, much faster than the relevant dynamical time scales. The SSX-FRC project therefore will provide the most detailed internal examination of FRC magnetic structure to date. Typical characteristics of SSX-FRC plasmas are 3-4 mWb poloidal flux, $1 \mathrm{kG}$ edge field, $1 \times 10^{15} / \mathrm{cm}^{3}$ density, $30 \mathrm{eV}$ temperature $\left(T_{e}+T_{i}\right)$, and $s>10$.

In this paper we describe the initial results from the SSX-FRC project, and it is organized as follows. Experimental details are described in the next section. Section III contains results of both co- and counter-helicity merging experiments, as well as initial results of dependence on RCC field strength. Observations and conclusions are summarized in Sec. IV.

\section{SSX-FRC EXPERIMENT}

The SSX-FRC apparatus uses opposing, coaxial magnetized plasma guns to produce spheromaks at either end of a cylindrical volume bounded by a thin shell copper flux conserver, as indicated in Fig. 1. The gun flux can be varied, but

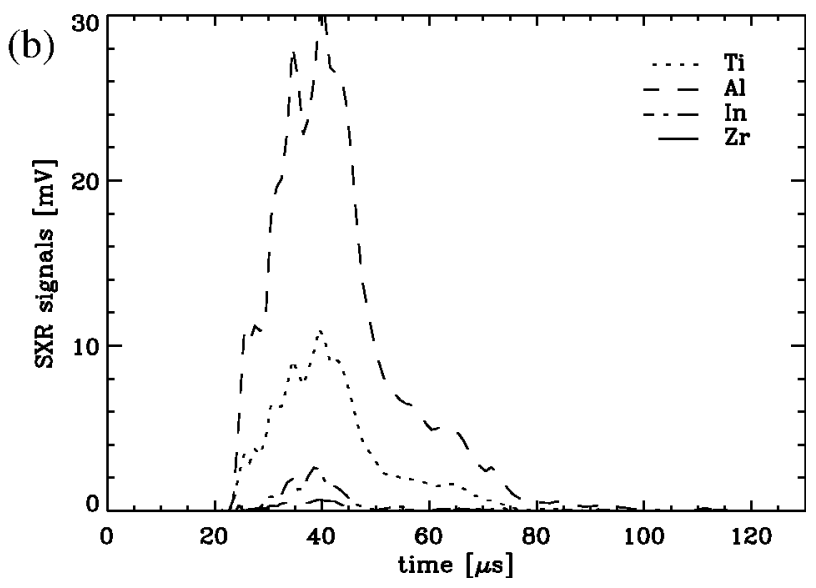

FIG. 2. Soft x-ray detector. (a) Spectral response functions for the unfiltered diode and four filters: $\mathrm{Al}, \mathrm{In}, \mathrm{Ti}, \mathrm{Zr}$. (b) Typical raw data for counter-helicity merging.

is normally operated at $1.0 \mathrm{mWb}$. For counter-helicity spheromak merging, the poloidal flux diverges from one gun and converges back in the other; the polarity of the flux in one of the guns is reversed to perform co-helicity merging experiments. Counter-helicity spheromaks at SSX-FRC therefore have parallel (attractive) toroidal currents, while co-helicity spheromaks have anti-parallel (repulsive) toroidal currents.

The poloidal flux surfaces depicted within the flux conserver boundary in Fig. 1 show the idealized configuration expected for modest (210 G on axis) RCC field strength should an equilibrium develop. These surfaces are calculated with a numerical Grad-Shafranov solver developed at General Atomics specifically for this project. The "doublet-CT" equilibrium has a high $\beta$ FRC region at the midplane confined only by a poloidal field that links private, unreconnected spheromak regions centered on two separate magnetic axes. Note also the region of good curvature at the midplane imposed by the RCC field shape. In order to maximize the opportunity to study FRC instabilities, the elongation selected for this experiment is deliberately MHD tilt unstable for a single axis CT.

The vacuum field from the two RCCs at the midplane are soaked through the flux conservers before the guns are fired. The separation of the RCC coils can be varied, but for initial experiments is fixed at $6.3 \mathrm{~cm}$. To maximize the field 

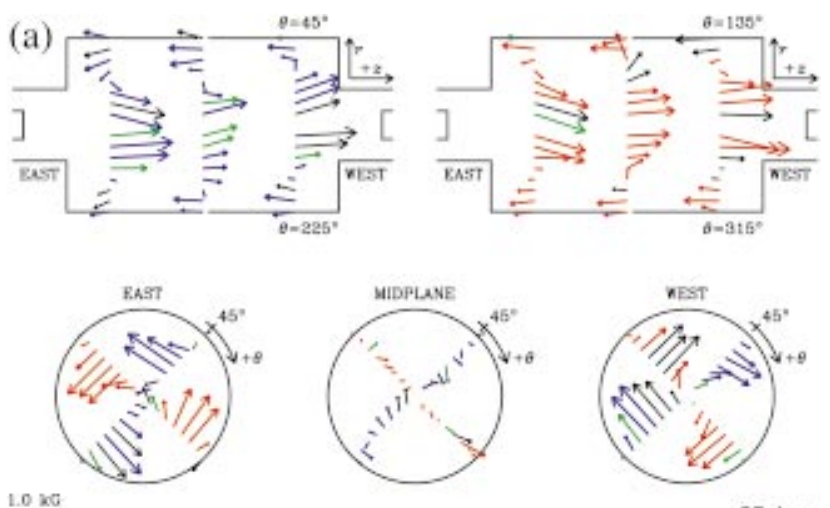

$\stackrel{1.0 \mathrm{NO}}{\longrightarrow}$

$62.4 \mu \mathrm{s}$
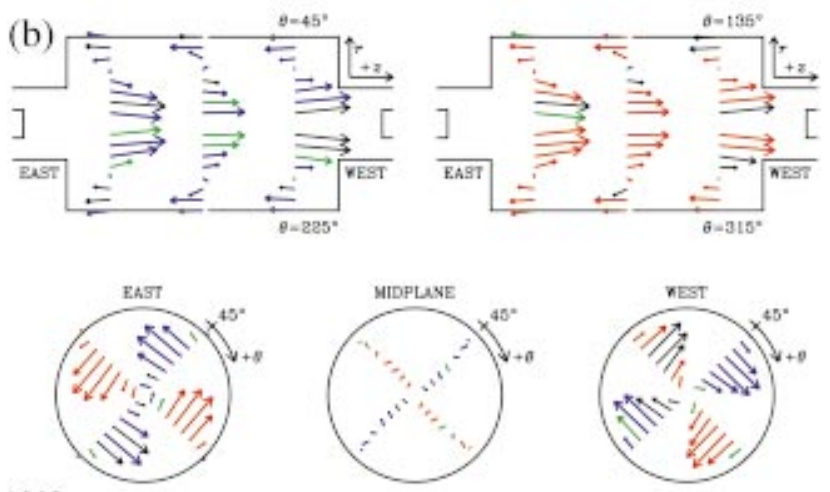

$\stackrel{t .0 \mathrm{~kg}}{\longrightarrow}$
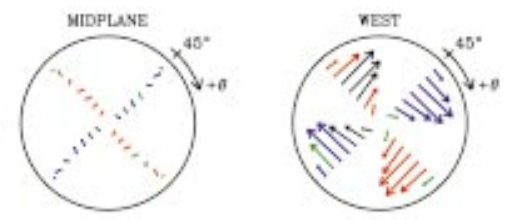

$62.4 \mu \mathrm{s}$

FIG. 3. (Color) Magnetic structure for a counter-helicity merging shot. The scale of a $1.0 \mathrm{kG}$ field is indicated at the bottom left, and the time at the bottom right. Five views of the data are shown: $r-\theta$ projections for the three axial locations (bottom row), and $r-z$ projections for the two possible poloidal cross-sections (top row, color coded red and blue). Black and green vectors indicate measurements where one or more of the coils in a triplet were broken. The flux-conserving boundary is shown in an outline for each view. (a) Full data. (b) $m=0$ (toroidal mode number) component.

curvature at the midplane, the RCCs are inside the vacuum chamber, as close as possible to the flux conservers, at an average diameter of $50 \mathrm{~cm}$. The field on axis from these coils can be set from zero to $750 \mathrm{G}$. The flux conserver diameter is $40 \mathrm{~cm}$. The total length between end wall plates of the flux conserver is $61 \mathrm{~cm}$, giving an elongation of 1.5 . There is a 2 $\mathrm{cm}$ gap between the two halves of the flux conserver to allow diagnostic access.

Linear magnetic probes with three-axis inductive loops at eight locations $(2.5 \mathrm{~cm}$ spacing) are inserted radially into the device at three axial positions, hereafter referred to as east, midplane, and west. There is access for probes to be inserted at up to eight equally spaced toroidal angles around the machine on the east and west ends. The midplane access is limited to four toroidal angles. The signals from the full set of probes (up to 600 coils) can be read out at $1.25 \mathrm{MHz}$ with a multiplexing data acquisition system. ${ }^{19}$ In the first set of SSX-FRC runs, only the four midplane linear magnetic probes were installed and the RCC field was scanned from zero to $700 \mathrm{G}$ in increments of $140 \mathrm{G}$. Subsequently, twelve probes were installed, four each in the east, midplane, and west, as shown in Fig. 1.

The diagnostic set also includes a He-Ne laser $(633 \mathrm{~nm})$ quadrature interferometer for electron density measurements,
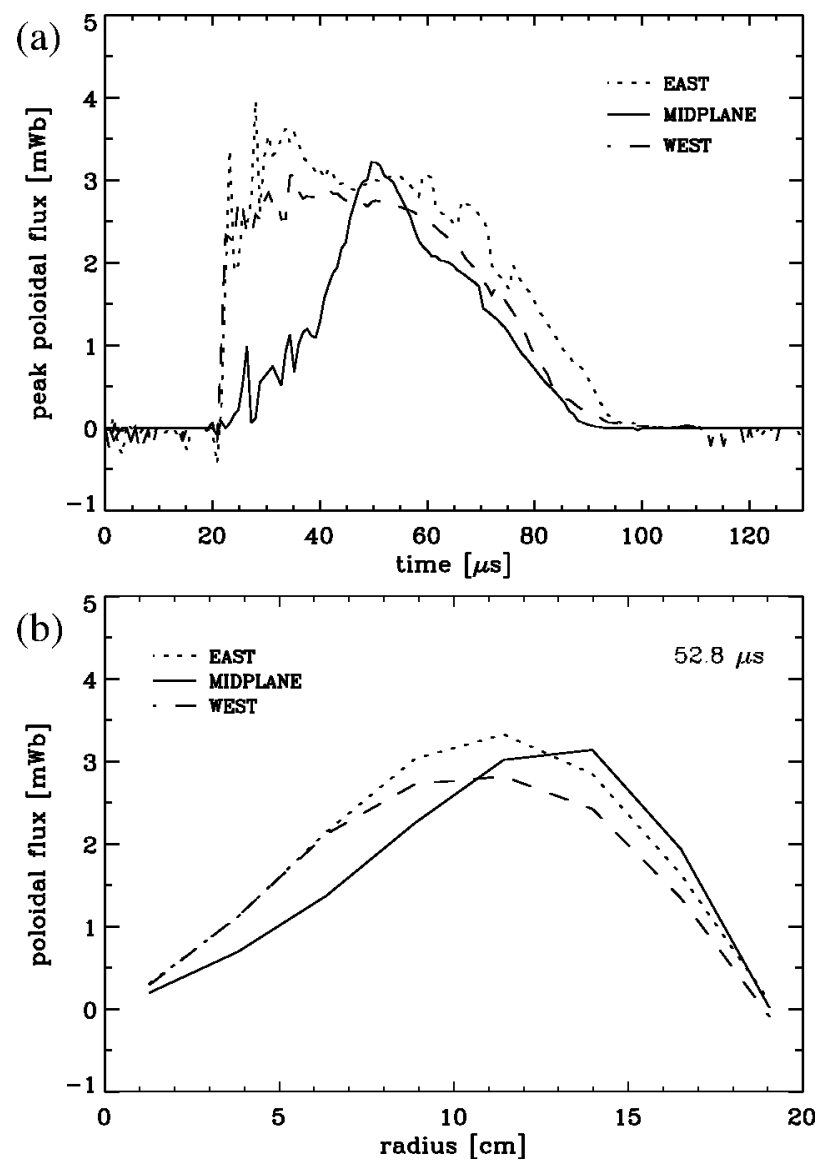

FIG. 4. Poloidal flux characteristics for a counter-helicity merging shot. (a) Time dependence of the peak poloidal flux; (b) radial flux profile at $t=52.8 \mu \mathrm{s}$.

a four channel soft $\mathrm{x}$-ray detector, ${ }^{20}$ and a six pin (Gundestrup) Mach probe. ${ }^{21}$ For the experiments described in this paper, the interferometer was set up to view a diameter on the east side of SSX-FRC, and the soft X-ray detector was placed at the midplane; the lines of sight are indicated in Fig. $1(\mathrm{~b})$.

The soft X-ray detector can be used to perform time dependent temperature measurements. A matched set of four PIN diodes is used (AXUV series from International Radiation Detectors) with a $0.1 \mu \mathrm{m}$ metal foil filter (Al, Zr, Ti, In) on each diode. The AXUV diodes have low noise and fast time response $(\leqslant 1 \mu \mathrm{s})$. Each diode sits behind a $0.5 \mathrm{~T}$ magnetic field from pairs of neodymium-iron-boron permanent magnets to suppress energetic ions. Figure 2 shows the spectral response of each filtered diode and sample signals. By fitting the set of diode signals to the convolution of the bremsstrahlung spectrum with each spectral response function, the electron temperature can be determined. A spectroscopic computation (SPEC3d) indicates that impurity radiation is a minimal contribution in the SSX-FRC temperature range $(10-100 \mathrm{eV})$ and impurity fractions $(<1-2 \% \mathrm{C}, \mathrm{N}$, O).

\section{RESULTS}

Figures 3, 4, 5, and 6 show data from a representative counter-helicity merging shot with an applied RCC field of 

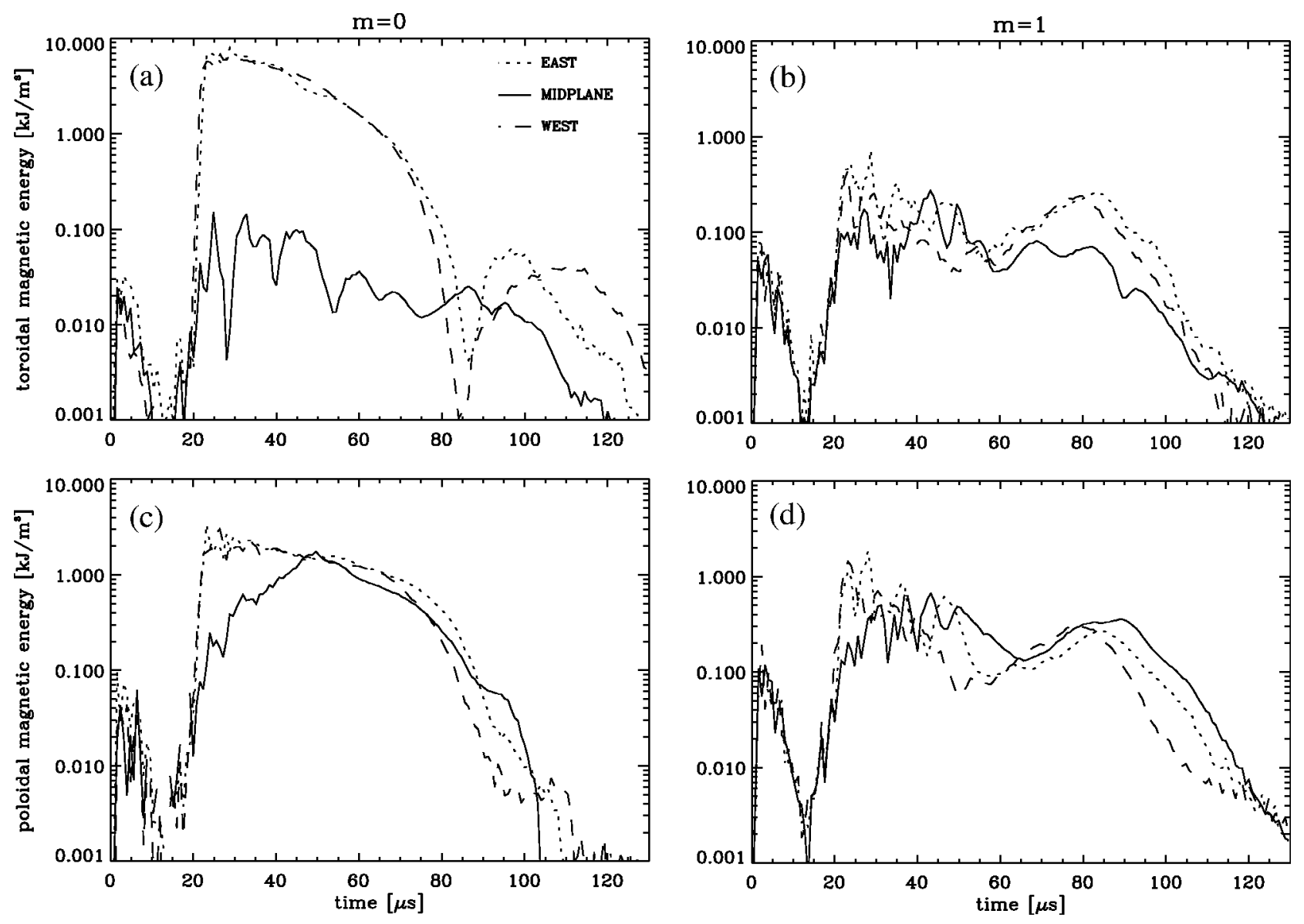

FIG. 5. Magnetic energy density dependence on time for a counter-helicity merging shot. (a) Toroidal $m=0$; (b) toroidal $m=1$; (c) poloidal $m=0$; (d) poloidal $m=1$.

$70 \mathrm{G}$ on axis. The vector plot of Fig. 3 depicts the magnetic structure at $t=62.4 \mu \mathrm{s}$ (the guns fire at $t \equiv 0 \mu \mathrm{s}$ and the spheromaks are ejected at $t \approx 20 \mu \mathrm{s})$, while Fig. 6 shows the typical structure late in time $(t=88 \mu \mathrm{s})$. Figures 4 and 5 indicate the time history of the poloidal flux and average magnetic energy densities in each axial plane for this run.

The toroidal resolution of the magnetic probe array in each $z$-plane permits Fourier decomposition into the toroidal $m=0$ and $m=1$ amplitudes of $B_{r}, B_{\theta}$, and $B_{z}$ at each of the eight radial probe positions. Flux is computed by integrating only the $m=0$ component of $B_{z}$, including the RCC field. Average magnetic energy densities are computed by integrating in $r$ and $\theta$ the sum of the squares of these mode amplitudes (Fourier orthogonality), using the measured radial dependence and the azimuthal dependence appropriate to each mode (constant in $\theta$ for $m=0$ and $\sin \theta$ and $\cos \theta$ for the two $m=1$ amplitudes). The vacuum energy density from the RCC is then subtracted.

The magnetic structure depicted in Fig. 3(a), and the symmetric $m=0$ component shown in Fig. 3(b) in particular, is similar to the doublet-CT equilibrium illustrated in Fig. 1. Reconnection has occurred, and the poloidal field is observed to link two bundles of toroidal flux concentrated at the east and west ends of the device. Very little toroidal field is measured at the midplane. Field reversal occurs in all three axial planes at $r \approx 13 \mathrm{~cm}$. Note that the data for the probes at the wall have not been corrected for the distortion caused by their proximity to the flux conserver. Comparing (a) and (b), the $m=0$ component dominates the magnetic structure at this time in the shot.

Figures 4 and 5 summarize the dynamics of this run. In Fig. 4(a) the poloidal flux on the east and west ends of the device reach a maximum of 3-4 mWb almost immediately after formation. This is consistent with the ejection of spheromaks from each gun with a flux amplification factor of $3-4$, and is typical of the gun formation method: as magnetofluid ejects from the gun, dynamo activity converts excess toroidal flux into poloidal flux to produce the equilibrated fields characteristic of a spheromak.

The rise of the poloidal flux at the midplane to the maximum at $t \approx 50 \mu$ s agrees with the picture of an increasing quantity of private flux in the two spheromaks reconnecting and joining the common flux. In this picture there are no private regions once all three planes have the same flux; nevertheless, Fig. 3 shows that toroidal field remains on each end with strength comparable to or greater than the poloidal field out to at least $t=62.4 \mu \mathrm{s}$. Rather than the doublet-CT, this is more like the structure of the Omelchenko FRC hybrid simulation ${ }^{14}$ described in the Introduction.

Figure 4(b) shows the flux profiles at the time of maximum midplane flux. The midplane profile peaks at a slightly larger radius, consistent with higher $\beta$ FRC structure, than at 

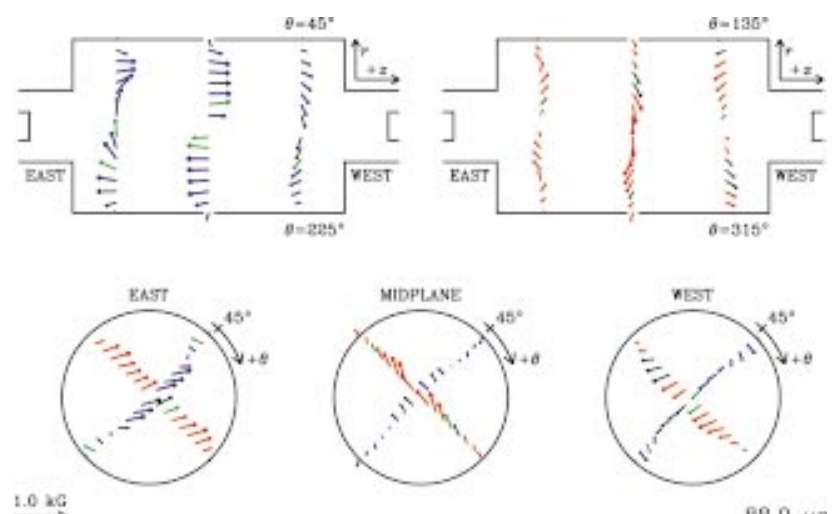

$88.0 \mu \mathrm{s}$

FIG. 6. (Color) The $m=1$ magnetic structure at $t=88 \mu$ s for a counterhelicity merging shot. This final state magnetic structure is consistent with a tilted CT.

the ends. In fact, the peak at $r=14 \mathrm{~cm}$ in the $R=20 \mathrm{~cm}$ flux conserver corresponds very well to the simple profileindependent pressure balance prediction $r=R_{s} / \sqrt{2}$ for a FRC. Furthermore, the peaks at $r=12 \mathrm{~cm}$ on the east and west ends are consistent with the spheromak prediction $r$ $\approx 0.62 R_{\mathrm{FC}}$ for the location of the peak poloidal flux. These observations imply a $\beta \approx 1$ FRC-like configuration at the midplane and a spheromak-like configuration at each end. At this time $t=52.8 \mu \mathrm{s}$ in the run, the edge field at the midplane is about $1.0 \mathrm{kG}$ (extrapolating to the flux conserver wall), and the density is approximately $1.0 \times 10^{15} / \mathrm{cm}^{3}$ (see below). Simple pressure balance therefore gives $T_{e}+T_{i} \approx 30 \mathrm{eV}$.

The $m=0$ and $m=1$ toroidal and poloidal average magnetic energy densities shown in Fig. 5 contain more information of the dynamic evolution of this run. The $m=0$ toroidal and poloidal energies on the east and west ends of the device are dominant until $t \approx 80 \mu \mathrm{s}$. At the midplane, the $m=0$ toroidal energy is insignificant for the duration of the run, as was seen at $t=62.4 \mu \mathrm{s}$ in Fig. 3(b). As the spheromaks merge, the midplane $m=0$ poloidal energy steadily increases until it reaches the same poloidal energy density as the ends at $t \approx 50 \mu \mathrm{s}$, in a manner similar to the increase of the midplane flux. Aside from the magnitude of the poloidal field, therefore, the magnetic structure during merging is similar to Fig. 3, as is verified by direct examination of the evolving vector plots of the magnetic structure.

The $m=1$ poloidal and toroidal energies during merging could come from turbulence in the merging process, asymmetric reconnection, or continued drive from the guns, and it is possible that there is strength in unresolved higher mode numbers. Whatever the source, the $m=1$ to $m=0$ poloidal energy ratio is initially about $30 \%$ and drops to about $10 \%$ once merging is complete at $t \approx 50 \mu \mathrm{s}$.

After $t \approx 50 \mu \mathrm{s}$, both the poloidal and toroidal $m=1$ energies show a remarkable period of exponential growth, with a characteristic time of $15-20 \mu \mathrm{s}$. After $t \approx 80 \mu \mathrm{s}$, the $m$ $=1$ poloidal and toroidal energies dominate the $m=0$ energies. Figure 6 shows a vector plot of the $m=1$ magnetic structure during this stage of the evolution of the run, at $t$ $=88 \mu \mathrm{s}$. This structure is consistent with a completely tilted CT: the magnetic axis is mostly aligned with the midplane
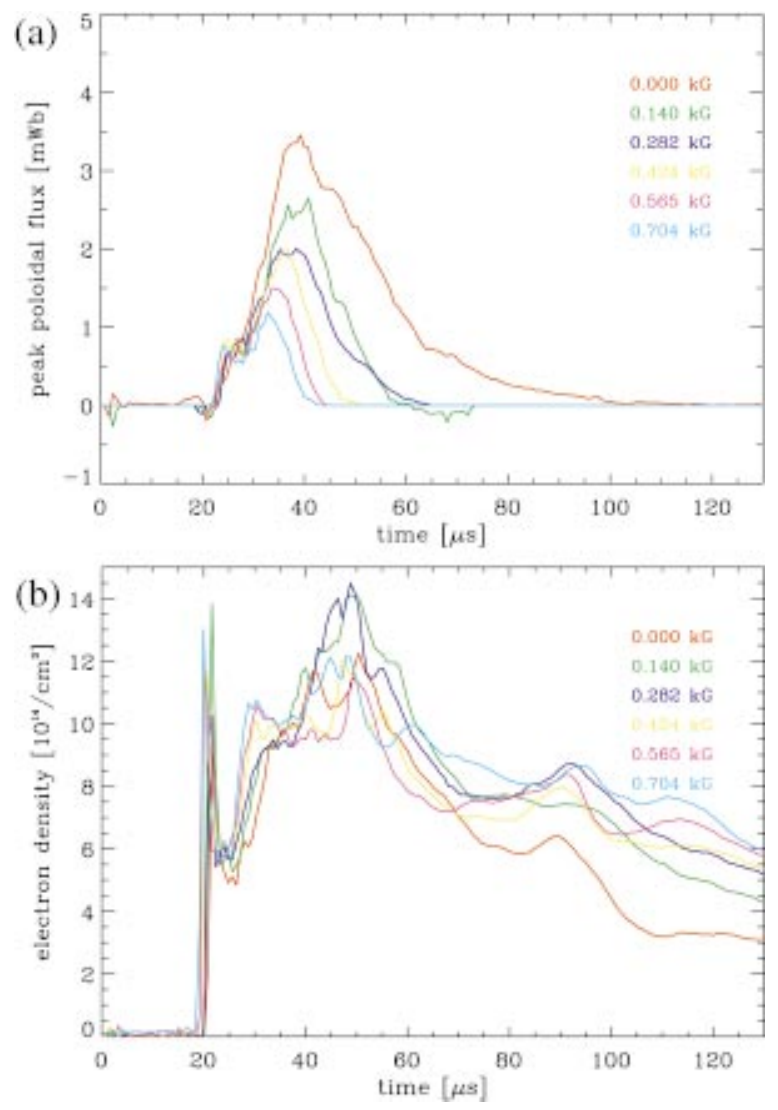

FIG. 7. (Color) RCC field strength dependences. (a) Peak midplane poloidal flux; (b) line averaged electron density.

probes at $\theta=135^{\circ}$ and $\theta=315^{\circ}$. The tilt instability therefore is most likely responsible for the growth of the $m=1$ poloidal energy observed after $t \approx 50 \mu \mathrm{s}$ in Fig. 5(d). Furthermore, a coherent tilt would also describe the rapid roll-off of the $m=0$ energy densities occurring just after the onset of the $m=1$ growth.

The growth rate of the tilt instability is often expressed in units of the axial MHD (Alfvén) transit time, $L / v_{\mathrm{A}}$, where $L$ is the separatrix half-length, and the Alfvén speed $v_{\mathrm{A}}$ is computed with the midplane edge field and density at the field null. At $t \approx 50-60 \mu \mathrm{s}$ when the $m=1$ poloidal energy begins to grow, the transit time is $L / v_{\mathrm{A}} \approx 5 \mu \mathrm{s}$, using $n$ $\approx 1.0 \times 10^{15} / \mathrm{cm}^{3}$ (see below). Therefore, recalling that the growth rate of the energy is twice that in the field, the observed tilt $e$-folds in 6-8 MHD times. In comparison, both MHD and kinetic calculations ${ }^{14,6}$ yield $e$-folding times of only about one MHD time for comparable FRC parameters.

Figure 7 summarizes the dependence of the poloidal flux and density on RCC field strength. This scan was performed with only the four midplane magnetic probes inserted into the machine. Figure 7(a) shows that the midplane poloidal flux generally survives longer at lower RCC strength. As expected, the quantity of poloidal flux decreases with increasing RCC strength, indicating that the merging process can be controlled. The higher RCC fields push the two CTs apart after their initial contact at the midplane. Aside from a mild increase at late times, the density does not vary significantly with RCC strength, as shown in Fig. 7(b). 

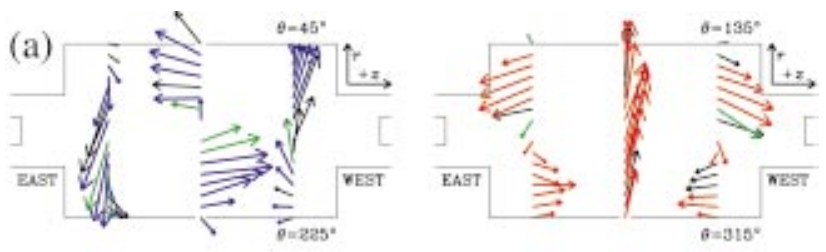

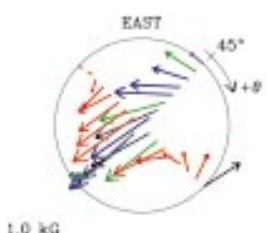

$\stackrel{1.0 \mathrm{~kg}}{\longrightarrow}$

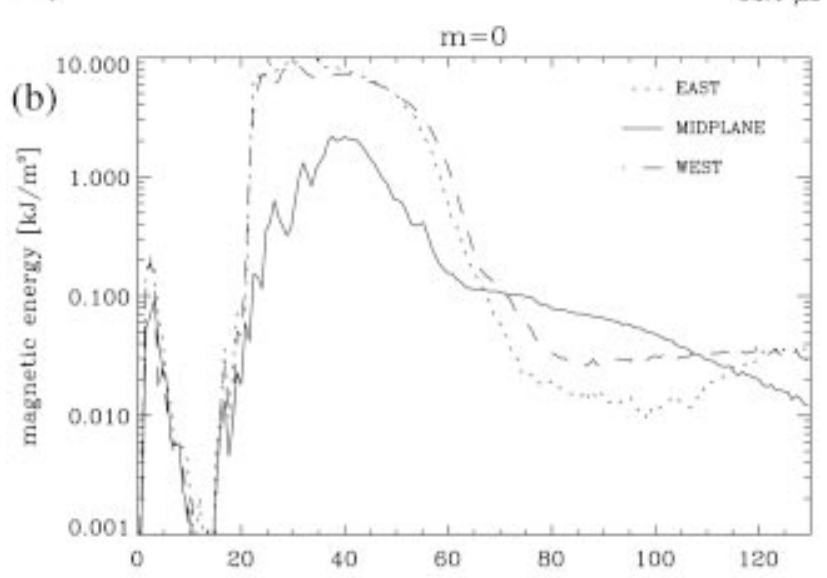

$m=1$

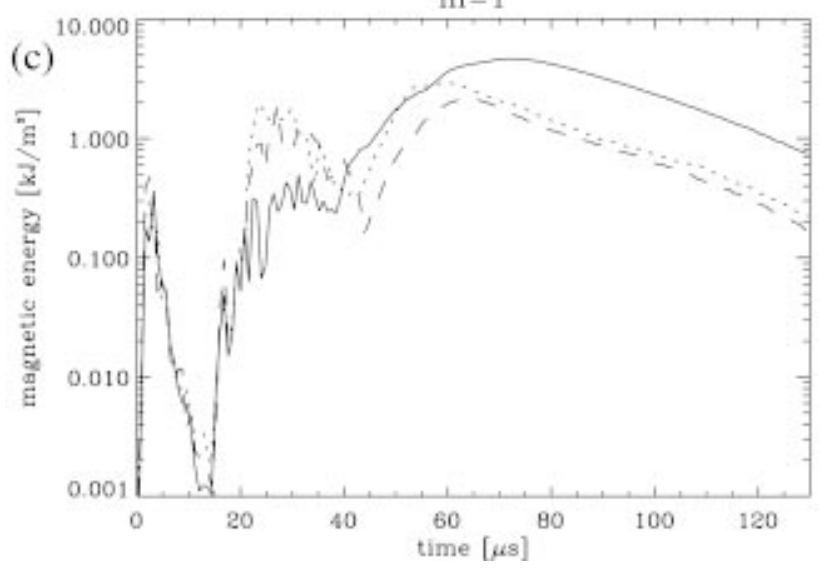

FIG. 8. (Color) Co-helicity merging. (a) Magnetic structure consistent with a tilted spheromak early in time $(t=62.4 \mu \mathrm{s})$; (b) $m=0$ energy density; (c) $m=1$ energy density.

In addition to counter-helicity merging, co-helicity merging runs have also been performed. By helicity conservation, co-helicity merging should form a single spheromak which, due to the aspect ratio of the flux conserver, will tilt. Magnetic data for a typical co-helicity run is displayed in Fig. 8. In fact, the structure shown in Fig. 8(a) is consistent with a tilted spheromak in a cylindrical flux conserver. ${ }^{22}$ For brevity, Figs. 8(b) and 8(c) shows just the total (sum of the poloidal and toroidal) $m=0$ and $m=1$ average magnetic energy densities for this run. Evidently, the tilt onset occurs much sooner and the $e$-folding time is much faster than for counter-helicity merging. Also, the tilted spheromak persists for an exceptionally long time, as can be seen in the time

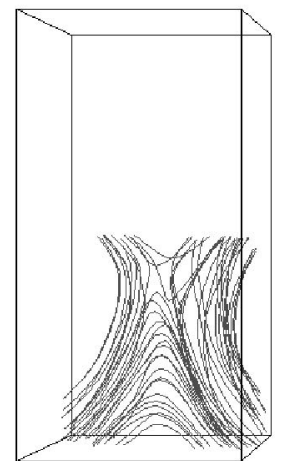

(a)

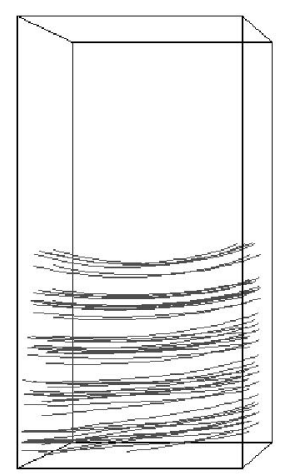

(b)
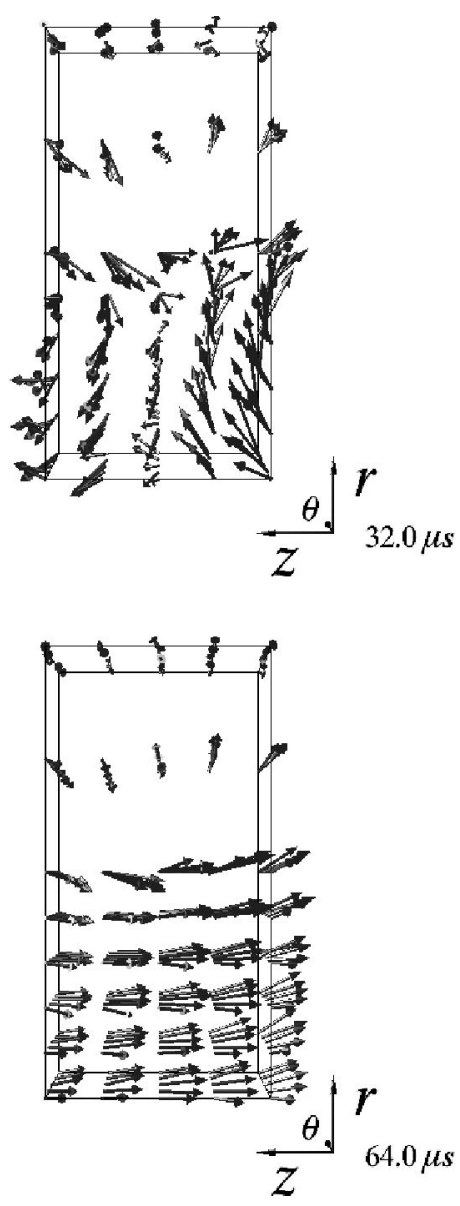

FIG. 9. Compact magnetic probe data ( $\approx 2 \mathrm{~cm}$ lattice) for a counter-helicity merging shot in $50 \mathrm{~cm}$ diameter flux conservers. (a) At $t=32 \mu \mathrm{s}$ reconnection is observed; (b) at $t=64 \mu \mathrm{s}$ the field structure is poloidal with no twist, consistent with a FRC. The last set of probes are at the flux conserver wall. Field lines at large $r$ curve to avoid $5 \mathrm{~cm}$ annuli.

dependence of the $m=1$ energy, compared to the counterhelicity case.

Finally, the local magnetic structure was studied during SSX-FRC prototyping experiments. The pre-existing ${ }^{1} 50 \mathrm{~cm}$ diameter SSX flux conservers were modified by cutting 40 $\mathrm{cm}$ diameter holes in the midplane walls. This left $5 \mathrm{~cm}$ wide annuli which were expected to act as passive RCCs. A 5 $\times 5 \times 8(2 \mathrm{~cm}$ lattice spacing) compact magnetic probe array was located at the midplane for these experiments. Figure 9(a) shows reconnection occurring at early times, just as the two counter-helicity spheromaks reach the midplane. Previous reconnection experiments at $\mathrm{SSX}^{16-18}$ have shown that this process has a unique three-dimensional character and can accelerate ions beyond the Alfvén energy. Later in time, the local field is very organized, with very little twist, as illustrated in Fig. 9(b), consistent with the dominantly poloidal fields observed in the midplane in Fig. 3. The effect of the $5 \mathrm{~cm}$ annuli can be seen in the curvature of the field lines at the largest values of $r$.

Reconnection during counter-helicity merging is known to be an ion heating mechanism. ${ }^{10}$ A comparison of the electron temperature (the thermal equilibration time between ions and electrons is estimated to be a few microseconds) obtained with the soft x-ray detector for co- and counter- 


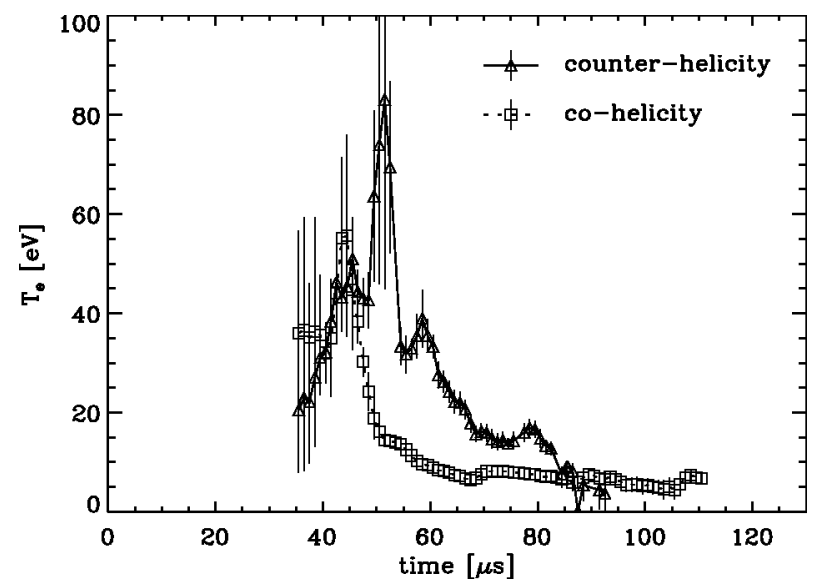

FIG. 10. Soft x-ray temperatures for co- and counter-helicity runs, computed assuming bremsstrahlung emission only (no impurity radiation). Counter-helicity runs have higher peak temperatures. The $\approx 5 \mathrm{eV}$ floor may be an artifact of the fitting process when the diode signals become small.

helicity merging is shown in Fig. 10. As expected, a much higher peak temperature is observed for counter-helicity merging than for co-helicity merging where reconnection does not occur. Note that the accuracy of the temperature, obtained assuming only bremsstrahlung, is less robust than the observation of a hotter plasma for counter-helicity merging.

\section{CONCLUSION}

In this paper we report the results from the first operation of SSX-FRC. A high $s$ FRC is produced by counter-helicity spheromak merging. The density reaches $1 \times 10^{15} / \mathrm{cm}^{3}$, and the temperature is estimated from pressure balance to be approximately $30 \mathrm{eV}$. Typical edge fields are $1.0 \mathrm{kG}$, and the null occurs near $r=13 \mathrm{~cm}$ in a $40 \mathrm{~cm}$ diameter flux conserver, giving $s>10$. The poloidal flux is typically 3-4 $\mathrm{mWb}$, corresponding to a factor of 3-4 amplification of the gun flux. The flux profile is consistent with $\beta \approx 1$ at the midplane. An axially antisymmetric toroidal field distribution is consistently observed. Even when the poloidal flux equalizes axially, indicating the presence of a single magnetic axis rather than a doublet-CT, the toroidal field does not annihilate. This behavior is not understood. Finally, toroidal mode analysis indicates that the final state is dominantly $m=1$ in character, and consistent with a completely tilted object. The growth time of the tilt instability is estimated to be $6-8$ MHD times. Co-helicity merging shows a more rapid evolution to a similar final state.

\section{ACKNOWLEDGMENTS}

Technical contributions from Steve Palmer and Jim Haldeman (Swarthmore) and Jim Leuer (GA), and discussions with Y. Ono (U. Tokyo) and P. Parks (GA) are gratefully acknowledged.

This work was supported by funding from Department of Energy (DOE) Grants No. DE-FG02-00ER54604, No. DE-FG02-00ER54654, and General Atomics.

${ }^{1}$ M. R. Brown, Phys. Plasmas 6, 1717 (1999).

${ }^{2}$ M. Tuszewski, Nucl. Fusion 28, 2033 (1988).

${ }^{3}$ J. T. Slough and A. L. Hoffman, Phys. Fluids B 5, 4366 (1993).

${ }^{4}$ M. Tuszewski, D. P. Taggart, R. E. Chrien, D. J. Rej, R. E. Siemon, and B.

L. Wright, Phys. Fluids B 3, 2856 (1991).

${ }^{5}$ J. T. Slough and A. L. Hoffman, Nucl. Fusion 28, 1121 (1988).

${ }^{6}$ E. Belova, S. C. Jardin, H. Ji, M. Yamada, and R. Kulsrud, Phys. Plasmas 7, 4996 (2000)

${ }^{7}$ J. H. Hammer, Nucl. Fusion 21, 488 (1981).

${ }^{8}$ M. N. Rosenbluth and M. N. Bussac, Nucl. Fusion 19, 489 (1979).

${ }^{9}$ Y. Ono, M. Inomoto, T. Okazaki, and Y. Ueda, Phys. Plasmas 4, 1953 (1997).

${ }^{10}$ Y. Ono, M. Yamada, T. Akao, T. Tajima, and R. Matsumoto, Phys. Rev. Lett. 76, 3328 (1996).

${ }^{11}$ Y. Ono, A. Morita, M. Katsurai, and M. Yamada, Phys. Fluids B 5, 3691 (1993).

${ }^{12}$ Y. Ono, M. Inomoto, Y. Ueda, T. Matsuyama, and T. Okazaki, Nucl. Fusion 39, 2001 (1999).

${ }^{13}$ M. Tuszewski and B. L. Wright, Phys. Rev. Lett. 63, 2236 (1989).

${ }^{14}$ Y. A. Omelchenko, M. J. Schaffer, and P. B. Parks, Phys. Plasmas 8, 4463 (2001).

${ }^{15}$ Y. A. Omelchenko, Phys. Plasmas 7, 1443 (2000).

${ }^{16}$ M. R. Brown, C. D. Cothran, M. Landreman, D. Schlossberg, and W. H. Matthaeus, Astrophys. J. Lett. 577, L63 (2002).

${ }^{17}$ M. R. Brown, C. D. Cothran, M. Landreman, D. Schlossberg, W. H. Matthaeus, G. Qin, V. S. Lukin, and T. Gray, Phys. Plasmas 9, 2077 (2002).

${ }^{18}$ T. W. Kornack, P. K. Sollins, and M. R. Brown, Phys. Rev. E 58, R36 (1998).

${ }^{19}$ M. Landreman, C. D. Cothran, M. R. Brown, M. Kostora, and J. T. Slough, Rev. Sci. Instrum. 74, 2361 (2003).

${ }^{20} \mathrm{~F}$. Hansen, Ph.D. thesis, Caltech 2001.

${ }^{21}$ S. Gerhardt, Ph.D. thesis, University of Wisconsin, 2002.

${ }^{22}$ T. R. Jarboe, I. Henins, H. W. Hoida, R. K. Linford, J. Marshall, D. A. Platts, and A. R. Sherwood, Phys. Rev. Lett. 45, 1264 (1980). 